\title{
Mid-infrared detector development for the Origins Space Telescope
}

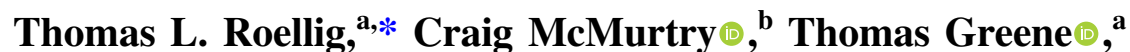 \\ Taro Matsuo $\odot$, ${ }^{\text {c Itsuki Sakon } \odot, ~}{ }^{\mathbf{d}}$ and Johannes Staguhn $\odot^{\text {e }}$ \\ ${ }^{a}$ NASA Ames Research Center, Space Sciences Division, Moffett Field, California, \\ United States \\ ${ }^{b}$ University of Rochester, Physics and Astronomy Department, Rochester, \\ New York, United States \\ 'Osaka University, Department of Earth and Space Science, Osaka, Japan \\ ${ }^{\mathrm{d}}$ University of Tokyo, Graduate Schools of Science, Department of Astronomy, Tokyo, Japan \\ ${ }^{\mathrm{e}}$ Johns Hopkins University, NASA/Goddard Space Flight Center, Department of Physics and \\ Astronomy, Greenbelt, Maryland, United States
}

\begin{abstract}
Mid-infrared detector arrays operating from 2.8 to $20 \mu \mathrm{m}$ are baselined in the design of the Origins Space Telescope Mid-Infrared Spectrometer Instrument. This instrument is designed to detect and measure the spectral signatures of gases of biogenic origin in the atmospheres of exoplanets as they transit their host stars. In order to make these detections, the detector array's pixels need to have high-signal stability when exposed to a constant flux in multiple timeseries integration over a typical transit time of a few hours. With the use of a densified pupil spectrometer design, pointing effects can be mitigated because pointing variations do not displace spectra on the detector and each wavelength of light is averaged over a large number of pixels, giving good spectrophotometric stabilities. The current state-of-the-art detector arrays do not achieve these stabilities, although with a feasible development program this level of performance should be achievable. Three detector technologies are under consideration for this development, $\mathrm{HgCdTe}$ arrays, Si:As impurity band conduction arrays, and transition edge superconductor bolometer arrays. We primarily treat the $\mathrm{HgCdTe}$ technology development, but also introduce the paths forward for the other two technologies. After a few years-long investigative programs, a down-select will be undertaken to select the flight technology. (C) The Authors. Published by SPIE under a Creative Commons Attribution 4.0 Unported License. Distribution or reproduction of this work in whole or in part requires full attribution of the original publication, including its DOI. [DOI: 10.1117/1.JATIS.6.4.041503]
\end{abstract}

Keywords: Origins; astronomy; detectors; infrared.

Paper 20076SS received Jun. 15, 2020; accepted for publication Dec. 1, 2020; published online Dec. 17, 2020.

\section{Introduction}

The Origins Space Telescope (Origins) traces our cosmic history, from the formation of the first galaxies and the rise of metals to the development of habitable worlds and present-day life. Origins does this through exquisite sensitivity to infrared radiation from ions, atoms, molecules, dust, water vapor and ice, and observations of extra-solar planetary atmospheres, protoplanetary disks, and large-area extragalactic fields. Origins operates in the wavelength range 2.8 to $588 \mu \mathrm{m}$ and is more than 1000 times more sensitive than its predecessors due to its large, cold (4.5 K) telescope and advanced instruments.

Studying the atmospheres of potentially habitable planets orbiting M-dwarf stars is a key science goal for the Origins Space Telescope. Transit spectroscopy has proven to be an essential tool for characterizing the atmospheres of exoplanets and the mid-infrared transit spectrometer instrument (MISC-T) on Origins will employ a densified pupil optical design, ${ }^{1}$ which, although limited in use for a wide range of astronomical investigations, is optimized for the removal of

*Address all correspondence to Thomas L. Roellig, Thomas.L.Roellig@nasa.gov 


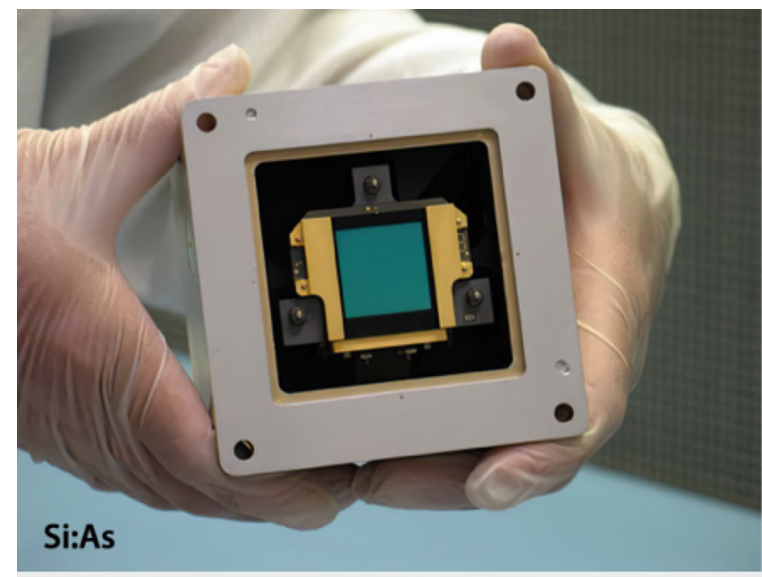

(a)

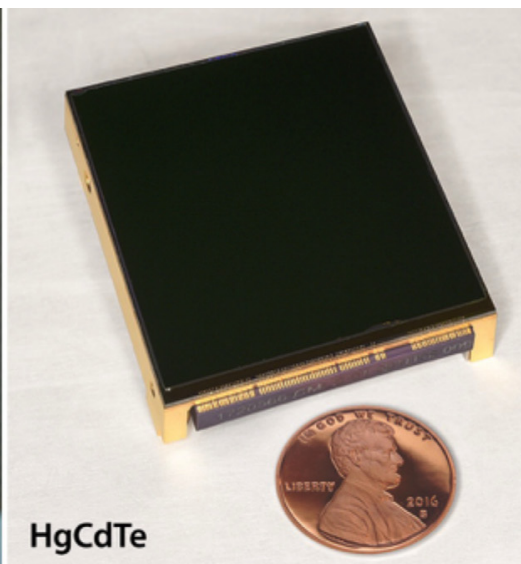

(b)

Fig. 1 State of the art mid-infrared detectors: (a) Si:As detector array used in the JWST/MIRI instrument and (b) HgCdTe long-wavelength detector $(\lambda>10 \mu \mathrm{m})$ to be used in the NEOSM.

Table 1 Detector requirements for the origins mid-infrared instrument (MISC-T).

\begin{tabular}{|c|c|c|c|c|c|}
\hline Inst. & $\lambda_{\min }(\mu \mathrm{m})$ & $\lambda_{\max }(\mu \mathrm{m})$ & $N_{\text {pix }}$ & Sensitivity & Saturation limits \\
\hline \multirow[t]{3}{*}{ MISC-T } & 2.8 & 20 & $10^{4}$ or $4 \times 10^{6}$ & $\mathrm{NEP}=3 \times 10^{-18}\left(\mathrm{~W} / \mathrm{Hz}^{1 / 2}\right)$ & $K \sim 3.0 \mathrm{mag}$ \\
\hline & & & & $\begin{array}{c}\text { DQE } 50 \% \text { to } 80 \% \text {, depending } \\
\text { on wavelength }\end{array}$ & $30 \mathrm{Jy}$ at $3.3 \mu \mathrm{m}$ \\
\hline & & & & $\begin{array}{c}5 \mathrm{ppm} \text { stability over a few hours } \\
\text { for the } 2.8-\text { to } 11-\mu \mathrm{m} \text { range, } \\
20 \mathrm{ppm} \text { for the } 11-\text { to } 20-\mu \mathrm{m} \text { range }\end{array}$ & $\begin{array}{l}\text { (Utilizing maximum } \\
\text { array read rate) }\end{array}$ \\
\hline
\end{tabular}

systematic instabilities in the detector arrays as well as due to pointing jitter and will achieve the necessary performance to detect and measure gases of biogenic origin in exoplanet atmospheres. The key performance metric for these observations is stability of the response of the instrument during the transit, secondary eclipse, or planetary orbit phase curve (typically on the time scale of hours to days). The James Webb Space Telescope (JWST) mid-infrared instrument (MIRI) uses the same detector technology as Spitzer/infrared array camera (IRAC) channels 3 and 4 [Fig. 1(a)], which have been able to achieve $\sim 60 \mathrm{ppm}$ precision on timescales of several hours, including the phenomological calibration of on-orbit drifts. Systematic errors for transit observations expected with JWST based on previous observations are described in Refs. 2 and 3.

The biosignature case for Origins requires a mid-IR detector stability of down to $\sim 5 \mathrm{ppm}$, depending on the wavelength, over several hours (Table 1). This stability requirement in the performance is an order of magnitude beyond the state-of-the-art that has been achieved on-orbit and may require new development beyond existing technology. Three detector technologies are under consideration for this development, $\mathrm{HgCdTe}$ arrays, Si:As impurity band conduction (IBC) arrays, and transition edge superconductor bolometer arrays. This paper primarily treats the $\mathrm{HgCdTe}$ technology development, but also introduces and compares the paths forward for the other two technologies.

\section{Current State-of-the-Art}

There have been remarkable recent achievements in stability with IR detectors. Knutson et al. ${ }^{4}$ obtained 65 ppm noise with Si:As arrays (similar to those on JWST) with Spitzer with relatively short integration times (Fig. 2). The InSb arrays on Spitzer have achieved 50 to 150 ppm stability, depending on the algorithm used to remove correlated noise. ${ }^{5,6}$ The Hubble Space Telescope

J. Astron. Telesc. Instrum. Syst. $\quad$ 041503-2 Oct-Dec 2020 • Vol. 6(4) 


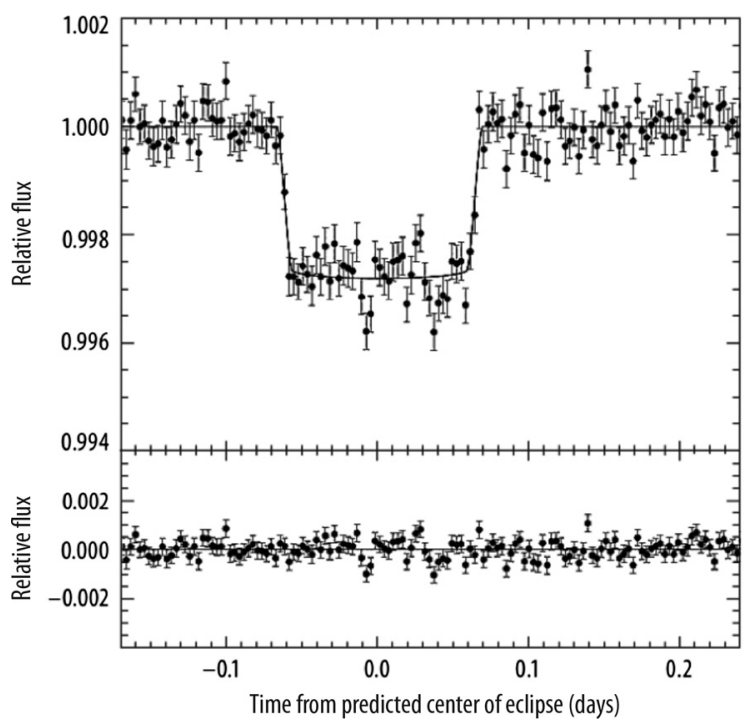

Fig. 2 Noise characteristics of exoplanet observations with Spitzer/IRAC. ${ }^{4}$ Figure 1 shows noise improving down to at least 50 ppm and perhaps even lower when binning multiple data points.

(HST) WFC3 with 1.7- $\mu \mathrm{m}$ H1RG HgCdTe arrays have achieved a full system stability of $23 \mathrm{ppm}$ in two exoplanet transits. ${ }^{7,8}$ JWST expects to achieve $\sim 20$ to $50 \mathrm{ppm}$ stability per observation with the H2RG 5- $\mu$ m cut-off arrays. ${ }^{2,9}$ The $\sim 50 \mathrm{ppm}$ stability for JWST is an "expected to achieve at least" level and is conservative given the difficulty in measuring such stabilities on the ground. JWST is likely to perform better than $50 \mathrm{ppm}$ once operational. The $23 \mathrm{ppm}$ from HST was a measured level for an on-orbit mission, i.e., lower noise environment than the ground.

JWST, Atmospheric Remote-sensing Infrared Exoplanet Large-survey (ARIEL), and the Transiting Exoplanet Survey Satellite will produce an extensive set of image processing and analysis algorithms, which can be used by Origins. In particular, JWST will observe spectral features in small transiting exoplanets with high precision over a large range of wavelengths, which will require processing at levels even beyond what has been done for Kepler, HST, and Spitzer. ${ }^{10}$ As for the long-wavelength performance of $\mathrm{HgCdTe}$ detectors, long-wavelength material has been developed for the Near Earth Object Surveillance Mission (NEOSM) that is sensitive out to $>10 \mu \mathrm{m}$. NEOSM is a wide-field survey mission, and as such it will not produce a data pipeline that is designed to look for exoplanets. However, NEOSM will publicly release large data sets that are time-series images. It is expected that the exoplanet community will produce data reduction software to use those data (albeit sparse for most exoplanet purposes).

The read noise of the Si:As detectors has not dramatically improved from the Spitzer/IRAC to JWST/MIRI (see Refs. 11 and 12, Figs. 14 and 11, respectively). With the read out integrated circuits (ROICs) used in Spitzer, the noise was largely unchanged as the temperature decreased below $40 \mathrm{~K}$. However, for JWST/MIRI prototype ROICs, the noise increased as the temperature decreased, ${ }^{11,13}$ which is due primarily to nonthermal and nonrandom noise components that do not reduce with either multiple sampling or multiple observations. The inverse temperature dependence of the read noise for the prototype ROICs over such a broad temperature range was not troubling since the MIRI arrays would be operated at a fixed temperature, but this behavior indicated where some of the problems originated in the ROIC design and fabrication. Subsequent improvements to the MIRI ROICs showed the read noise was about $14 \mathrm{e}^{-12,14}$ for multiple sampling (Fowler-8). However, that is very similar to the read noise achieved for Spitzer IRAC Channel $3,{ }^{15}$ which is 9 to $11 \mathrm{e}^{-}$for Fowler-16 sampling with 12 - and 30 -s integration, respectively, i.e., square root of 2 difference as expected for the Fowler-8 versus Fowler-16 sampling. Many researchers are currently working to improve effective stability through image processing, but that is an ex post facto solution. However, much can be done to improve the stability while the data are being taken (i.e., improve the technology as well as the image processing software). It should be pointed out that read noise should not be an issue if it is random 
because Origins observations should be source-noise limited on bright host stars. Systematic noise (e.g., correlation between pixels) is another story-it will not go down with more photons.

Spitzer/IRAC Si:As detectors have demonstrated $\sim 60 \mathrm{ppm}$ precision in transit observations of several hours. JWST/MIRI is expected to achieve similar or slightly better stability (e.g., Refs. 3 and 16), with the limiting factors in stability currently unidentified. Once on orbit, the MIRI detectors are expected to establish the current state-of-the-art for transit spectroscopy in the mid-infrared band. MIRI uses the same arsenic-doped silicon IBC detector technology as Spitzer/IRAC and a hybridized readout that improves their stability. Improvements can definitely be made-current laboratory testing employing an engineering pathfinder for the JWST Si:As arrays and a readout averaging scheme as used in a densified pupil spectrometer design has given stabilities that are over a factor of four better than achieved with the Spitzer IRAC detectors + telescope system performance. ${ }^{17}$

\section{Mid-IR Array Challenges}

The biggest challenge to reaching Origins' mid-infrared requirements involves the stability requirement for the transit spectrometer instrument. It is likely there are a number of factors (e.g., pointing jitter and control electronics limiting the stability of the Spitzer and JWST observations). Unfortunately, laboratory systems simulating end-to-end observations do not currently provide the required stability to measure at the levels needed to simulate even Spitzer or JWST observations. A few teams (Staguhn, Greene, and Matsuo) are currently working to improve the laboratory systems to improve ground-testing capabilities.

Because high-stability detectors are critical to the success of the MISC transit channel, we envision a dedicated detector program to identify a detector technology that meets the stability requirements. We expect that once such a detector is identified, that an appropriate instrument would be designed based on the detector. Sections 3.1, 3.2, and 3.4 outline the steps needed to progress $\mathrm{HgCdTe}, \mathrm{Si}: \mathrm{As}$, and transition edge sensors (TES) detector technologies to meet the highest precision Origins MISC-T detector requirements (given in the second column of Table 2). Section 3.3 discusses how to reinstitute the production of Si:As detectors. In Sec. 4, we describe the strategy for mid-infrared array development and a down-select strategy.

Table 2 Origins detector requirements and current $\mathrm{HgCdTe}$ state-of-the-art.

\begin{tabular}{lcc}
\hline \hline Parameter & Origins needs/requirements & HgCdTe state-of-the-art \\
\hline $\begin{array}{l}\text { Format } \\
\text { Pixel size }(\mu \mathrm{m})\end{array}$ & $2 \mathrm{k} \times 2 \mathrm{k}$ pixels & $2 \mathrm{k} \times 2 \mathrm{k}(2040 \times 2040$ active) pixels \\
Wavelength coverage $(\mu \mathrm{m})$ & 2.8 to 11 & 18 \\
Readout rate & 10 s full frame & 16 \\
DQE & $>50 \%$ at all wavelengths in & 1.3 s full frame (32 outputs)
\end{tabular}




\subsection{HgCdTe Detector Array Technology Development}

Mid-IR detector stability was recently added to the NASA Science Missions Directorate Astrophysics Division "Technology Gap List" in recognition of the importance of exoplanet characterization and the power of the mid-IR to trace important molecules in the atmospheres of exoplanets discovered through transit observations. ${ }^{18}$ The Origins team has assessed that the mid-IR HgCdTe detector arrays, developed with Teledyne Imaging Sensors under several NASA grants to the University of Rochester (UR), are a better alternative than Si:As IBC arrays for sensitive exoplanet characterization missions requiring system stabilities of $5 \mathrm{ppm}$ at wavelengths $<11 \mu \mathrm{m}$. The 3- to $11-\mu \mathrm{m}$ range is better covered by HgCdTe than Si:As, since at short wavelengths, Si:As is known to be semitransparent. For example, the stratospheric observatory for infrared astronomy faint object infrared camera for the SOFIA telescope instrument with Spitzer-era Si:As detectors had a detective quantum efficiency (DQE) of $\sim 20 \%$ at $4 \mu \mathrm{m},{ }^{19}$ while for the $\mathrm{HgCdTe}$ arrays on JWST, the corresponding number at this wavelength is $\sim 90 \%{ }^{20}$ As Refs. 21 and 13, specifically in Fig. 5, have shown, Si:As detector arrays are quite transparent below $10 \mu \mathrm{m}$. Photons that are not absorbed in the detector material can either (1) produce a diffraction pattern in reflection off of the metal pads ${ }^{22}$ or (2) enter and bounce around in the layer between the detector and ROIC material before being reflected back into Si:As detector. If epoxy is used to fill the gap between the detector material and the ROIC, this will produce a wavelength dependence to these features that corresponds to absorption by the epoxy. In addition, $\mathrm{HgCdTe}$ photodiodes do not exhibit gain variation with bias, whereas Si:As IBCs do. HgCdTe photodiodes operate at a higher temperature - above the mobility freeze-out temperature in $\mathrm{Si}$, which allows for a more standard fabrication of their ROICs and will simplify the cooling requirements of the detector arrays. These reasons point to better performance at lower operational (observing efficiency) and mission cost with $\mathrm{HgCdTe}$ arrays for the shorter $(<11 \mu \mathrm{m})$ wavelengths.

If JWST/NIRCam or NIRSpec perform at the 5-ppm system level, those techniques and software can be implemented on Origins. In the intervening years before Origins is built and launched, Origins will improve the detector technology, ROIC, and control electronics.

\subsubsection{HgCdTe detector development plan}

To make HgCdTe viable for use on Origins will require ROIC stability improvements, including major development programs that address decreasing read noise, alleviating nonlinearity effects, reducing pixel-to-pixel variations, and resetting anomalies. The remaining challenges are to: (1) increase the full well, which may require a selectable capacitor/pixel addition to the ROIC, which is fairly straight forward and (2) redesign the ROIC to decrease read noise and change pixel size while not increasing dark current or adversely affecting the image quality [modulation transfer function (MTF) or point spread function (PSF)]. The second challenge can be investigated and worked out with multiple production iterations (see task 4).

$\mathrm{HgCdTe}$ has benefitted from existing development work for JWST (5.0 $\mu \mathrm{m}$ cutoff), NEOSM (10 $\mu \mathrm{m}$ cutoff), and UR's $15-\mu \mathrm{m}$ cutoff development aimed at supporting spectroscopic detection of biosignatures via space missions, such as Origins. (The ARIEL mission will also require similar detectors to those on NEOSM.) For the past three years, UR has been extending this technology to longer wavelength cutoffs. The results for $13-\mu \mathrm{m}$ cutoff wavelength detector arrays were very promising with dark currents, well depths, and operabilities ${ }^{23-25}$ similar to those of the earlier $10-\mu \mathrm{m}$ cutoff wavelength detector array produced for NEOSM. For the 15- to $16-\mu \mathrm{m}$ cutoff wavelength detector arrays produced in the second half of the UR development program, ${ }^{26}$ the dark currents were higher and the well depths were lower than Origins requirements. However, those requirements are set by the needed sensitivity at shorter wavelengths, i.e., it would be possible to use $\mathrm{HgCdTe}$ arrays to cover from 2.8 to $16 \mu \mathrm{m}$ using two or three cut-off wavelength arrays, e.g., $6,11.5$, and 16- $\mu \mathrm{m}$ cut-off wavelength detector arrays, and still meet Origins requirements at each of those bands.

The growth of $\mathrm{HgCdTe}$ detectors is often fraught with difficulties, e.g., low well depth and high dark current due to tunneling currents, softer material at longer wavelengths, which makes hybridization more difficult, cluster defects such as those from $\mathrm{Hg}$ precipitates, thinning of substrates, and targeting the cut-off wavelength. Although each of these issues may be understood in

J. Astron. Telesc. Instrum. Syst. $\quad 041503-5 \quad$ Oct-Dec 2020 • Vol. 6(4) 
principle, it is difficult to fully constrain all of them in practice, which results in lower yield of detectors, which in turn, results in higher costs to produce the flight detectors for any given program. Taking what is learned during both JWST's and NEOSM's production runs of flight detector arrays (heritage reduces cost and risk) will aid in improving detector yield.

The next steps are to address the remaining requirements for Origins: stability, read noise, and well capacity. At low enough temperatures ( $35 \mathrm{~K}$ for $10-\mu \mathrm{m}$ cutoff and $27 \mathrm{~K}$ for $13-\mu \mathrm{m}$ cutoff), the dark currents are bias dependent, i.e., over the course of an integration, charge accumulation will cause the dark current to decrease. This is an inherent instability in that kind of dark current. One possibility is that dark current stability can be reached by operating at a sufficiently high temperature $(28 \mathrm{~K})$ so the detector is thermally dark current limited $(\mathrm{G}-\mathrm{R})$ instead of bias-dependent dark current limited (trap-assisted tunneling), while still meeting the $0.3 \mathrm{e}^{-} / \mathrm{s}$ dark current requirement for Origins. It will need to verify whether or not a more stable but slightly higher dark current is capable of producing better overall stability as compared to the instabilities due to the bias-dependent dark current. Teledyne HxRG ROICs, generally used for low background space astronomy, are source follower per detector pixel and the lowest noise and low-power choice. Although a capacitive transimpedance amplifier capacitive transimpedance amplifier ROIC could provide constant bias, which might initially seem the most stable choice, other reasons (e.g., high power-typically Watts rather than milliwatts-and glow) make this ROIC an unsuitable choice for cryogenic temperature space missions, at least as they are currently envisioned. The high power occurs because all pixels are powered during the read of each pixel. Some detector groups have discussed turning off the power to all but the pixel being read out; however, then turning on the power to each pixel would be unstable and still generate more power than a few milliwatts. Extra closed cycle cryo-refrigerators would be required, adding cost and weight to the mission. The high current would also lead to ROIC glow, which affects the background flux levels in an unstable way. The most stable performance from arrays comes with not undergoing any changes from integration to integration (including keeping the same background power and signal position on the detector, which is exactly how exoplanet transit observations are done). First efforts will be directed toward improving the HxRG ROICs before considering a completely new cryogenic ROIC design.

Redesigning the ROIC will, by definition, lower the complete detector array's technology readiness level (TRL) to 4. Once the design is complete and tested in a lab environment, it will be back at TRL 5. We expect that the primary need for retesting will be susceptibility to cosmic ray damage, as $\mathrm{HgCdTe}$ detector material is susceptible to damage from cosmic rays. The current $10-\mu \mathrm{m}$ cutoff $\mathrm{HgCdTe}$ arrays have been qualified to TRL 6 for the NEOSM mission after proton irradiation experiments where $<1 \%$ of the pixels were degraded (increased dark current) after $1.5 \times$ and $2.0 \times$ lifetime total ionizing does for the L1 environment. ${ }^{27}$ The HAWAII-2RG HgCdTe arrays have been qualified to $>$ TRL6 for JWST, OCO-2, HST, and ECHO. ${ }^{28}$ The redesigned detector array/ROIC will then need to pass proton radiation testing, thermal cycling, and vibration testing to once again reach TRL 6 . With sufficient funding, this process will take $\sim 4$ to 5 years.

\subsubsection{HgCdTe detector manufacturing}

The primary objective is to improve the overall radiometric stability of the $\mathrm{HgCdTe}$ detector arrays. This requires several tasks, as detailed below.

Task 1. The most important objective is to decrease the noise, both the long-term (between exposures taken over one day) $1 / f$ noise component and the short-term (single image) nonrandom components of the read noise. The $1 / f$ noise component directly impacts the long-term radiometric stability since it adds uncertainty to the measurement that cannot be removed via repeated sampling to reduce the noise. Popcorn (random telegraph) noise is another form of both traditional read noise and $1 / f$ noise that follows a power law distribution in both frequency of transitions and voltage of transitions. The lowest frequency popcorn noise has the largest voltage transition. There are a few methods to reduce $1 / f$ noise. First, the use of buried channel metal oxide silicon field effect transistor (MOSFETs) can reduce the $1 / f$ and popcorn noises. Second, switching to an all P-channel metal-oxide-semiconductor (PMOS) process [i.e., not fully complementary metal-oxide-semiconductor (CMOS)] can reduce the number of defects introduced during processing, i.e., fewer dopants and thus fewer contaminants entering the silicon.

J. Astron. Telesc. Instrum. Syst. $\quad$ 041503-6 Oct-Dec 2020 • Vol. 6(4) 
The process itself could be cleaned to the point of removing the contaminant responsible for the popcorn noise. Unless the read noise is very large, exoplanet transit observations will be photon shot noise limited due to bright host stars in the raw data.

The source-follower FET has a thermal shot-noise component due to the current that flows in the FET. That thermal noise is white across frequencies and thus may be reduced by decreasing the bandwidth of the FET. The reduction of the bandwidth is achievable via increasing the number of ROIC outputs while decreasing the rate with which each pixel is read, i.e., $20 \mu \mathrm{s} /$ pixel instead of 10. This option increases the power consumption per array due to the increased number of outputs, however, that is still within our budget of $100 \mathrm{~mW}$ per array. This option is unlikely to be needed since the thermal shot noise will of the ROIC will be reducible with multiple sampling and will be well below the photon shot noise of the individual observations.

Both the dopant and concentration of the dopant in the silicon wafer can be adjusted to improve the cryogenic performance of the ROIC. ${ }^{29}$ In addition, it is possible to improve the foundry process to make each layer more planar, i.e., place more oxide between FETs and traces such that the overlying oxide on those traces is flat and plane parallel with the starting silicon wafer. Without such a process improvement, the oxide becomes thinner at the corners of the traces (as seen in cross section) and leads to microshorts between crossing traces or the substrate. ${ }^{30}$ Adjustments to the combined parameters of the insulating oxide thickness, its planarization, the dopant, and its concentration will help to reduce microshorts within the ROIC, which, in turn, will reduce currents in the substrate, thereby reducing clock feed-through, which is a nonrandom component of the read noise.

Task 2. Implement design improvements to improve the stability of clocks and biases, which, in turn, will improve the overall detector stability. This is primarily a strict analysis of the design used to reduce stray capacitances and any potential shorts or crosstalk between adjacent circuits, e.g., move shift registers away from sensitive bias lines and output amplifiers (source follower FETs), adding more metal layers for shielding, employing lower voltages for switches, and dual polarity (differential) switches. The designs will also need to minimize the resistance of long lines for voltage supplies to decrease the voltage drop that occurs from one side of the ROIC to the other. The increase in pixel size will provide the ability to reduce resistance on long supply lines (reset voltage and drain voltage) using larger width traces, while also spacing those traces better to decrease the amount of stray capacitance. The improvements that were introduced into the HAWAII-4RG ROIC design to minimize clock feed-through will also be utilized in these designs. Finally, this task period will also cover optimizing the external electronics, such as voltage supplies and preamps, whose performance will also be critical in achieving the required detector stability.

Task 3: Improve the pixel-to-pixel uniformity. An increase in pixel size will allow for better pixel-to-pixel uniformity since the FETs will be well above the design rule limits for the chosen foundry, and thus inherent foundry process variations in the FET dimensions will be a smaller fraction of the area of the FETs. It should be noted that the densified pupil design also mitigates the requirement of better pixel-to-pixel uniformity because the densified pupil spectrum always forms on the same position of the detector as long as the target object is in the field of view of the MISC-T instrument.

Task 4 . Change the pixel pitch from $18 \mu \mathrm{m}$ to an optimized value derived by the final optical design of the MISC-T instrument. The existing HAWAII-2RG $2 \mathrm{k} \times 2 \mathrm{k}$ pixel ROICs used for $\mathrm{HgCdTe}$ arrays have $18-\mu \mathrm{m}$ pixel pitches, which may not be optimum-for example, pitches that are approximately diffraction-sized do not produce good image quality (e.g., MTF ${ }^{31}$ ). Further, increasing the size of the pixel pitch will allow more room within each pixel to accommodate changes made to satisfy the other tasks, both above and below.

Increasing the pixel pitch is not without its disadvantages. The final ROIC, produced after Origins selects its optimal design, will be large in physical area, and therefore, require stitching of reticles during the foundry process in order to create the full array. The optics for any future telescope will be more difficult to design given that the array extends over a larger area of the focal plane, thus putting more strain on the optical designer to deliver a flat focus over that larger area, although it should be noted that slower optical systems are generally less susceptible to aberrations. Furthermore, the size of the actual detector die will cover a larger portion of the $\mathrm{HgCdTe}$ wafers, which results in greater risk of nonuniformity of the detector and thus impacts the yield. With a larger detector die to be hybridized to the ROIC comes a larger force needed to 
bond all the pixels. This will require a short development period in order to be able to reproducibly hybridize the larger $\mathrm{HgCdTe}$ die to the larger ROIC die.

A pixel pitch increase would normally increase the dark current, i.e., larger $\mathrm{p}-\mathrm{n}$ junctions for larger pixels. However, the size of the $\mathrm{p}-\mathrm{n}$ junction needs not change, hence, dark current will not change. Despite having smaller $\mathrm{p}-\mathrm{n}$ junctions relative to the pixel area, a pixel pitch increase will not adversely affect the lateral charge collection by a large amount due to Teledyne's proprietary techniques in growing the $\mathrm{HgCdTe}$. The MTF will degrade slightly for shorter wavelength light $(<6 \mu \mathrm{m})$ due to the larger path lengths for diffusion. However, for wavelengths $>6 \mu \mathrm{m}$, the MTF will actually improve for larger pixel spacing due to the fact that the pixel size will be larger than the wavelengths of the collected photons, minimizing diffraction effects.

The interpixel capacitance (IPC) will also be affected by the change in pixel size. However, an increase in pitch will result in a decrease in IPC, which is a beneficial outcome, and an increase in the pixel pitch would allow more room to accommodate an increase in the capacitance allowing a commensurate increase in well depth as described in Task 5.

All of these advantages and disadvantages will need to be properly weighed during a trade study on the pixel size. Although the current goal is to produce $15-\mu \mathrm{m}$ pixels (as given by the optics team to the detector team), it is likely that Origins will decide to use pixels that are larger than that.

Task 5. Add (switchable if needed) internal capacitance to each unit cell (pixel) to increase the well capacity and reduce the bias dependence of DQE and dark current by effectively reducing the contribution of the detector to the total capacitance. This unfortunately will also increase the effective read noise since the read noise is actually a voltage noise despite always being quoted in electrons. The increased read noise must be traded against the gain in well capacity in order to find the optimal value for the added internal capacitors.

Task 6. Once a final ROIC design is made, it can be qualified to TRL-6. Part of that qualification process involves proton radiation testing, which can be conducted on bare ROICs.

Task 7 . The final objective is to produce hybridized long wavelength infrared (11- $\mu \mathrm{m}$ cutoff) $\mathrm{HgCdTe}$ detector arrays utilizing the best ROIC design. These arrays can then be tested against the Origins requirements. A small number of these arrays will be subjected again to radiation testing, thermal cycling, and vibe/shake testing to complete the full array qualification to TRL-6.

\subsection{Si:As Detector Development Plan}

The Si:As detectors that are currently baselined in the design of the MISC-T instrument longwavelength channel will operate over 11 to $20 \mu \mathrm{m}$, where this detector material has high quantum efficiency. The cooling requirements are very modest as these detectors will operate quite happily at the temperature of the Origins optics ( $\sim 6 \mathrm{~K}$, similar to the temperature of the JWST/ MIRI detectors ${ }^{32}$ ) and their power dissipation is low enough that simple heat straps will be sufficient to cool the detectors. Most importantly, the stability requirements are considerably relaxed compared to the shorter wavelengths - the biogenic spectral features of interest are much more pronounced at these longer wavelengths. As a result, there is little additional development that is needed to meet these requirements. The detector format specified in the MISC-T design $(2 \mathrm{k} \times 2 \mathrm{k})$ have four times the number of pixels as the $1 \mathrm{k} \times 1 \mathrm{k}$ Si:As detectors used in the JWST MIRI instrument, but increasing the pixel count is expected to be straightforward. These detectors are, therefore, already at a TRL 6 level for their stability, and at TRL 4 considering the need to increase the pixel count.

The biggest issue is the question of detector availability. Industrial detector fabricators (Raytheon and DRS) have built these detectors in the past for such missions as Spitzer, the Wide-field Infrared Survey Explorer (WISE) mission, and JWST. Raytheon has dropped their device fabrication of this technology, but DRS is still working on Si:As and Si:Sb devices for the space infrared telescope for cosmology and astrophysics (SPICA) mission. Unfortunately, SPICA is not a selected mission yet, and as such very little money has been sent to DRS for work on either Si:As or Si:Sb arrays [private communication with DRS and Takehiko Wada, Japanese Space Agency (JAXA)]. In fact, the last successful growth for Si:As was in 2012. Since then very little has been done due to lack of funding. The past developments were largely funded by interest from the Department of Defense, which is no longer interested in this

J. Astron. Telesc. Instrum. Syst. $\quad$ 041503-8 Oct-Dec 2020 • Vol. 6(4) 
wavelength range. As a result, the industrial fabrication facilities and institutional experience have declined significantly. Rebuilding this capability will be costly and it may be difficult to restart the equipment and reassemble experienced staff.

The development programs for both the ROIC and the detector material will run in parallel, where the final stage of both programs will be merged to produce a $2 \mathrm{k} \times 2 \mathrm{k} \mathrm{Si}$ :As array that will meet the requirements of Origins.

\subsection{HgCdTe and Si:As Detector Testing}

NASA Ames Research Center (ARC) and UR have been conducting tests of mid-IR array detectors for many years and have supported detector testing of a number of earlier and current space missions, including Spitzer, WISE, NEOSM, and JWST. The ARC facilities are currently in use to test the stability and performance of the densified pupil spectrometer design ${ }^{17}$ being considered for Origins. The UR facilities are currently in use to test the preflight production of $\mathrm{HgCdTe}$ arrays for NEOSM. To fully test the detector/spectrometer designs, both the ARC and UR facilities must be upgraded to measure the extremely low (few ppm over $10 \mathrm{~h}$ ) pixel-to-pixel photometric stabilities necessary to meet Origins transit science requirements, including the effects of flight-like disturbances. These facilities must be capable of testing the Si:As and $\mathrm{HgCdTe}$ detector technologies currently baselined for the Origin mid-IR instrument, which, in practice, means separate cryogenic Dewars will be required for each detector type. The scope of these test facility improvements can be completed in time to support the TRL5 demonstration by 2025, and TRL 6 detector technologies by Origins preliminary design review, currently anticipated to occur in 2027.

\subsection{TES Development Plan}

The Origins team is also studying TES ${ }^{33}$ as an alternative option for the transit spectrometer. This alternative option for the mid-infrared detectors will leverage the TES development effort that is being undertaken for the Origins, Origins Survey Spectrometer, and far-infrared imager and polarimeter instruments. ${ }^{34,35}$ To provide a detector array with the required stability while reducing the development efforts, the team selected an extremely stable and linear superconducting detector, TES, in combination with a calibration system. There are two disadvantages of this technology compared to the large-format $\mathrm{HgCdTe}$ and $\mathrm{Si}$ As detectors: (1) as their array sizes are smaller than the $2 \mathrm{k} \times 2 \mathrm{k}$ sizes used in the MISC-T densified pupil optical design, using TES detectors would necessitate a completely different optical design, and (2) they would require additional cryocoolers within the MISC-T instrument in order to reach their sub-Kelvin operating temperatures. However, in spite of these disadvantages, we feel that pursuing this alternative technology development path is an important risk-reduction approach to achieving the very high detector stability requirements. Given the impact on the MISC-T optical and mechanical designs and the additional cryogenic requirements for the TES detectors, the selection of which detector technology to use will have to be made before the Origins System Requirements Review.

Large-format, high-efficiency, low-noise TES-based bolometer arrays operating in the millimeter through far-infrared wavelength regimes have already been developed. ${ }^{36}$ Such arrays have been produced at NASA GSFC and successfully integrated into bolometer cameras [e.g., goddard-IRAM superconducting 2-millimeter observer (GISMO) and high-resolution airborne wideband camera plus (HAWC+)]. These detectors have very low $1 / f$ noise with $1 / f$ knees of close to $0.01 \mathrm{~Hz}$, as shown in a lab current noise density measurement of a Primordial Inflation Polarization Explorer (PIPER) balloon instrument array (Fig. 3). The adiabatic demagnetization refrigerator used to cool the detector was running open loop during the measurements; with feedback, the device will be even more stable.

The PIPER detectors have sufficient sensitivity and dynamic range for mid-IR spectroscopic applications with an noise equivalent power (NEP) of a few $10^{-18} \mathrm{~W} / \sqrt{\mathrm{Hz}}$ and a saturation power of $\sim 2 \mathrm{pW}$. Since TES detectors are used as bolometers (resistance changes with temperature), they can also be used as near- and mid-IR detectors. The only required development for this application is a suitable mid-infrared absorber to deposit the radiation energy into the detector membrane. The required absorbers can be optimized independently of other detector parameters such that a high quantum efficiency can be achieved. 


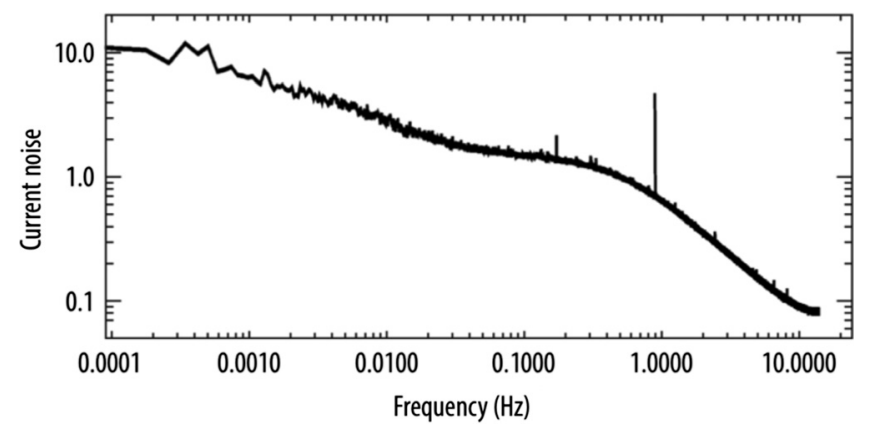

Fig. 3 Long duration laboratory measurement of the average noise spectrum of a GSFC TES detector array (in units of normalized current noise density).

To extend stability of the mid-IR TES arrays to the required time scales required for the Origins application (i.e., hours), the Goddard Space Flight Center (GSFC) team introduced a calibration system with a high-precision blackbody reference load and real-time load temperature monitoring. ${ }^{37}$ The load temperature is modulated, so detector response to the load signal can be monitored. The Origins planned observation targets (M-stars with and without planet) are always unresolved, i.e., point sources. The simultaneous illumination pattern of the detectors by the modulated blackbody source will not vary, and therefore, the relative pixel-pixel spectral response of the detectors can be cross calibrated so that of the spectral variations the M-stars can be measured to the required accuracy.

The blackbody temperature measurement can be done to the required sensitivity of $5 \mathrm{ppm}$ for the MIR spectral region within $100 \mathrm{~s}$ or less because the measurement itself is being obtained in the Wien side of the BB spectrum in the visible by a photodiode, where only 35-ppm precision is required to obtain the needed signal-to-noise ratio (SNR). During a 100-s integration time, the requirement for stability of the detectors is significantly looser than $5 \mathrm{ppm}$, simply due to the noise properties of the observed targets, the most extreme of them being the nearest target, Proxima Centauri. The SNR implies only a required stability of $\sim 10^{-3}$ within a second, resulting in a stability requirement of $\sim 10^{-4}$ in $100 \mathrm{~s}$ for the TES detectors. TES have been demonstrated to have a $1 / f$ knee longward of $100 \mathrm{~s}$, and the stability requirements over the many hours transit durations can be met as shown in Ref. 38.

The calibration scheme is independent of the detector used (even though it will only provide a sufficient SNR for calibrating individual time intervals of several minutes, so the detector needs to be stable within a fraction of that, which was demonstrated for TES). Using this calibration scheme with other detector, technologies such as those described in Secs. 3.1 and 3.2 would also be straightforward. This system approach has recently been awarded funding under NASA's Astrophysics Research and Analysis program.

\section{Schedule for MIR Array Development and Down-Select Strategy}

A dedicated mid-infrared detector program for Origins' MISC-T instrument will provide a strategic NASA investment outside of the grants program. This program will focus on the development of a high-stability mid-infrared transit spectrometer. In Sec. 3, we outlined the efforts needed to advance HgCdTe arrays, Si:As arrays, and TES bolometers. Each of these technologies is close to meeting the requirements, so it is quite likely that at least one will have demonstrated the needed performance by the time of the mission Preliminary Design Review. By the start of this development program (2021), JWST will be taking data and we will have better data to evaluate the performance of the $\mathrm{HgCdTe}$ and $\mathrm{Si}$ :As arrays and their applicability to Origins transit spectrometer. Hence, within the first year, it will be possible to assess the best path to take. For example, all options of $\mathrm{HgCdTe}$, Si:As, and/or TES bolometers could be pursued.

If all options are pursued, the strategy will focus on maximizing resources by implementing a step-wise review and progress assessment program, so that a technology that meets and shows performance needed for Origins will be chosen as early as possible. The Origins team envisions 
any mid-IR detector development will be in collaboration between industry, NASA centers, and universities. A rough estimated cost to mature the Origins mid-infrared detector technology is \$28 M (in Real Year dollars, based on informal conversations with IR detector vendors).

\section{Acknowledgments}

To enable the community to prepare for the 2020 Decadal Survey, NASA sponsored studies of four large mission concepts, of which Origins was one. NASA's Goddard Space Flight Center (GSFC), NASA Ames Research Center (ARC), the Japanese space agency, JAXA, Osaka University, and the Northrup Grumman and Ball Aerospace corporations all contributed substantial additional labor and financial support.

\section{References}

1. I. Sakon et al., "Mid-Infrared Spectrometer Camera (MISC) baseline and upscope," J. Astron. Telesc. Instrum. Syst., (2020).

2. C. Beichman et al., "Observations of transiting exoplanets with the James Webb Space Telescope (JWST)," Publ. Astron. Soc. Pac. 126(946), 1134 (2014).

3. T. P. Greene et al., "Characterizing transiting exoplanet atmospheres with JWST," Astrophys. J. 817, 17 (2016).

4. H. A. Knutson et al., "The 8 micron phase variation of the hot Saturn HD 149026b," Astrophys. J. 703, 769 (2009).

5. J. G. Ingalls et al., "Repeatability and accuracy of exoplanet eclipse depths measured with post-cryogenic Spitzer," Astron. J. 152, 44 (2016).

6. B-O. Demory et al, "Detection of thermal emission from a Super-Earth," Astrophys. J. 751, 28 (2012).

7. H. A. Knutson et al., "Hubble Space Telescope near-IR transmission spectroscopy of the Super-Earth HD 97658b," Astrophys. J. 794, 155 (2014).

8. L. Kreidberg et al., "A detection of water in the transmission spectrum of the hot Jupiter WASP-12b and implications for its atmospheric composition," Astrophys. J. 814, 66. (2015).

9. C. Clanton et al., "Precision near-infrared photometry for exoplanet transit observations. I. Ensemble spot photometry for an all-sky survey," Publ. Astron. Soc. Pac. 124, 700 (2012).

10. "JWST Approved GTO Programs page," https://www.stsci.edu/jwst/observing-programs/ approved-gto-programs.

11. C. W. McMurtry, W. J. Forrest, and J. L. Pipher, "James Webb space telescope: noise results for the mutliplexers of the mid-infrared instrument (MIRI)," Proc. SPIE 5902, 590208 (2005).

12. N. A. Lum et al., "Low-noise, low-temperature $256 \times 256$ Si:As IBC staring FPA," Proc. SPIE 1946, 1-10 (1993).

13. G. H. Rieke et al., "The mid-infrared instrument for the James Webb Space Telescope, VII: the MIRI detectors," Publ. Astron. Soc. Pac. 127, 665 (2015).

14. M. Ressler et al., "Performance of the JWST/MIRI Si:As detectors," Proc. SPIE 7021, 702100 (2008).

15. "IRAC Instrument Handbook," Spitzer Documentation \& Tools, https://irsa.ipac.caltech .edu/data/SPITZER/docs/irac/iracinstrumenthandbook/7/ (2015).

16. P. J. Love et al., " $1024 \times 1024$ Si: As IBC detector arrays for JWST MIRI," Proc. SPIE 5902, 590209 (2005).

17. T. Matsuo et al. "Photometric precision of a Si:As impurity band conduction mid-infrared detector and application to transit spectroscopy," Publ. Astron. Soc. Pac. 131(1006), 124502 (2019).

18. "Technology needs and gap lists," https://exoplanets.nasa.gov/exep/technology/gap-lists/ (2019).

19. L. D. Keller et al., "FORCAST: a facility 5-40 micron camera for SOFIA," Proc. SPIE 4014, 86-97 (2000). 
20. J. Garnett et al., " $2 \mathrm{k} \times 2 \mathrm{k}$ molecular beam epitaxy $\mathrm{HgCdTe}$ detectors for the James Webb Space Telescope NIRCam instrument," Proc. SPIE 5499, 35-46 (2004).

21. J. L. Pipher et al., "Comparison of laboratory and in-flight performance of infrared array camera (IRAC) detector arrays on Spitzer Space Telescope," Proc. SPIE 5487, 234 (2004).

22. G. H. Rieke, private communication (2019).

23. W. J. Forrest et al., "Development of megapixel $\mathrm{HgCdTe}$ detector arrays with 15 micron cutoff," in AAS Meeting \#228, 216.18 (2016).

24. C. W. McMurtry et al., " 13 micron cutoff $\mathrm{HgCdTe}$ detector arrays for space and groundbased astronomy," Proc. SPIE 9915, 99150E (2016).

25. M. S. Cabrera et al., "Development of 13 micron cutoff $\mathrm{HgCdTe}$ detector arrays for astronomy," J. Astron. Telesc. Instrum. Syst. 5(3), 036005 (2019).

26. M. S. Cabrera, "Development of a 15 micron cutoff $\mathrm{HgCdTe}$ detector array for astronomy," J. Astron. Telesc. Instrum. Syst. 6(1), 011004 (2019).

27. M. L. Dorn et al., "Proto irradiation results for long-wave $\mathrm{HgCdTe}$ infrared detector arrays for Near-Earth Object Camera," J. Astron. Telesc. Instrum. Syst. 2, 036002 (2016).

28. M. E. McKelvey et al., "Radiation environment performance of JWST prototype FPAs," Proc. SPIE 5167, 223-234 (2004).

29. C. W. McMurtry et al., "Next generation space telescope: NIR InSb array development," Proc. SPIE 4850, 847-857 (2003).

30. A. Hoffman, private communication (2003).

31. G. D. Boreman, Modulation Transfer Function in Optical and Electro-Optical Systems, SPIE Press, Bellingham, Washington (2001).

32. M. E. Ressler et al., "The mid-infrared instrument for the James Webb Space Telescope, VIII: the MIRI focal plane system," Publ. Astron. Soc. Pac. 127(953), 675 (2015).

33. J. E. Sadleir et al., "The state-of-the-art for TESS in the Origins band," J. Astron. Telesc. Instrum. Syst., (2020).

34. M. Bradford et al., "The Origins Survey Spectrometer (OSS): revealing the hearts of distant galaxies and forming planetary systems with ultrasensitive far-IR spectroscopy," J. Astron. Telesc. Instrum. Syst., (2020).

35. J. Staguhn et al., "Far-infrared Imager and Polarimeter (FIP)," J. Astron. Telesc. Instrum. Syst., (2020).

36. D. J. Benford et al., " 5,120 superconducting bolometers for the PIPER balloon-borne CMB polarization experiment," Proc. SPIE 7741, 77411Q (2010).

37. J. Staguhn et al., "An ultra-stable mid-infrared sensor for the detection of bio-signatures by means of transit spectroscopy," in The Space Astrophysics Landscape for the 2020s and Beyond, Proc. Conf. held 1-3 April, 2019 in Potomac, Maryland, LPI Contribution No. 2135, id.5029 (2019).

38. J. Staguhn et al., "An ultra-stable mid-infrared sensor for the detection of bio-signatures by means of transit spectroscopy," in IEEE Aerosp. Conf., Big Sky, Montana (2019).

Thomas L. Roellig has worked in the NASA civil service as an astrophysicist at the NASA Ames Research Center since 1980 and is currently the chief of the Astrophysics Branch at Ames. His scientific research interests have spanned a wide range of infrared astronomy and astronomical instrumentation development. He has conducted research and published papers in infrared instrument development, solar science, solar system science, star formation, interstellar medium, and brown dwarf astronomy.

Craig McMurtry: Biography is not available.

Thomas Greene received his $\mathrm{PhD}$ in astronomy from the University of Arizona in 1991 and held positions at the University of Hawaii Institute for Astronomy and Lockheed Martin before joining NASA in 1998. He is an astrophysicist at NASA's Ames Research Center in Mountain View, California. He is a co-investigator on the JWST NIRCam instrument and a member of the JWST Mid-Infrared Science Team and conducts observational studies of young stars and extrasolar planets. 
Taro Matsuo is an associate professor of physics at Nagoya University. His research is focused on the field of exoplanet science, with an emphasis on developing several instrument concepts for characterization of nearby habitable planet candidates, including transit spectrographs, interferometers, and coronagraphs.

Itsuki Sakon received his BS and MS degrees from the University of Tokyo of Astronomy in 2003 and 2005, respectively, and his PhD in science from the University of Tokyo in 2008. He is an assistant professor at the University of Tokyo. His current research interests include midinfrared astronomical instrumentation development as well as infrared observational astronomy, laboratory astrophysics, and astrochemistry.

Johannes Staguhn received his $\mathrm{PhD}$ in physics from the University of Cologne, Germany. He joined NASA/GSFC in 2000, working on far-infrared instrumentation and astronomy. $\mathrm{He}$ is a principal research scientist at Johns Hopkins University. He is a principal investigator of the bolometer camera GISMO and deputy study scientist and instrument scientist for the Origins Space Telescope. Currently, he is leading the development of a mission concept for the characterization of the atmospheres of planets around M-stars. 